\title{
CONSTRUÇÃO E VALIDAÇÃO DA ESCALA "AVALIAÇÃO DA COMPREENSÃO DO PACIENTE SOBRE ORIENTAÇÕES DE ALTA"
}

Rednaj Jaure Bacury Souza Mota ${ }^{1}$ Ana Claudia Giesbrecht Puggina ${ }^{2}$
ORCID: https://orcid.org/0000-0002-1680-8448

ORCID: https://orcid.org/0000-0001-8095-6560

Objetivo: Construir e investigar as propriedades psicométricas de uma escala que avalia a compreensão do paciente sobre orientações de alta. Método: Estudo metodológico quantitativo realizado com 72 pacientes e dois observadores. A validação foi obtida por meio da análise fatorial e correlação intra-classe. A consistência interna foi verificada pelo Alpha de Cronbach. Resultados: A escala foi construída em duas partes: a parte I (14 itens) refere-se a compreensão referida e é respondida pelo paciente, a parte II (8 itens) refere-se a compreensão percebida e é respondida por um observador. A consistência interna foi considerada excelente $(\alpha=0.918)$ na parte I e aceitável na parte II, tanto para observador $1(\alpha=0.724)$ quanto para o observador $2(\alpha=0.749)$. A reprodutibilidade inter-observador foi aceitável $(r=0.824)$. A análise fatorial da parte I determinou quatro fatores: (1) compreensão sobre medicamentos; (2) compreensão sobre tratamento e diagnóstico; (3) compreensão sobre restrição e (4) compreensão sobre retorno. Conclusão: A escala "Avaliação da compreensão do paciente sobre orientações de alta" foi considerada confiável e válida.

Descritores: Comunicação Não Verbal; Estudos de Validação; Alta do Paciente.

\section{CONSTRUCTION AND VALIDATION OF THE SCALE "ASSESSMENT OF PATIENT COMPREHENSION OF DISCHARGE INSTRUCTIONS"}

Objective: To construct and evaluate the psychometric properties of a scale that assesses patient understanding of discharge guidelines. Method: A quantitative methodological study involving 72 patients and two observers was conducted. Validation was obtained through factor analysis and intra-class correlation. Cronbach's alpha coefficient was used to measure internal consistency. Results: The scale consisted of two parts: part I (14 items) refers to the reported comprehension and is answered by the patient; part II (8 items) refers to the perceived comprehension and is answered by an observer. Internal consistency was excellent in part I $(\alpha=0.918)$ and acceptable in part II for both observer $I(\alpha=0.724)$ and observer $2(\alpha=0.749)$. Interobserver reproducibility was acceptable $(r=0.824)$. Factor analysis of part I determined four factors: 1) understanding of medications, 2) understanding of treatment and diagnosis; 3) understanding of restrictions, and 4) understanding of return.

Conclusion: The scale "Assessment of patient comprehension of discharge instructions" was considered reliable and valid.

Descriptors: Nonverbal Communication; Validation Studies; Patient discharge.

\section{CONSTRUCCIÓN Y VALIDACIÓN DE LA ESCALA "EVALUACIÓN DE LA COMPRENSIÓN DEL PACIENTE SOBRE ORIENTACIONES DE ALTA"}

Objectivo: Construir y investigar las propiedades psicométricas de una escala que evalúa la comprensión del paciente de las pautas de alta. Metodo: estudio metodológico cuantitativo realizado con 72 pacientes y dos observadores. La validación se obtuvo mediante análisis factorial y correlación intra-clase. La consistencia interna fue verificada por el Alpha de Cronbach. Resultados: La escala fue construida en dos partes: la parte I (14 ítems) se refiere a la comprensión referida y es respondida por el paciente, la parte II (8 ítems) se refiere a la comprensión percibida y es respondida por un observador. La consistencia interna fue considerada excelente $(\alpha=0.918)$ en la parte I y aceptable en la parte II tanto para el observador 1 ( $\alpha=0.724)$ como para el observador $2(\alpha=0.749)$. La reproducibilidad inter-observador fue aceptable ( $r=0.824)$. El análisis factorial de la Parte I determinó cuatro factores: (1) la comprensión de los medicamentos; (2) comprensión sobre tratamiento y diagnóstico; (3) comprensión sobre restricción y (4) comprensión sobre retorno. Conclusión: La escala "Evaluación de la Comprensión del Paciente sobre Orientaciones de Alta" fue considerada confiable y válida.

Descriptores: Comunicación No Verbal; Estudios de Validación; Alta del Paciente.

1Secretaria de Estado de Saúde do Amazonas, AM ${ }^{2}$ Faculdade de Medicina de Jundiaí, São Paulo, SP

Autor correspondente: Ana Claudia Giesbrecht Puggina E-mail: claudiagpuggina@gmail.com

Recebido: 11/10/2019

Aceiro: 10/12/2019 


\section{INTRODUÇÃO}

Os problemas relacionados à segurança do paciente no ambiente hospitalar têm levado as organizações de saúde no mundo a investigar as causas, visando conscientizar os profissionais e propor medidas para reduzir os riscos de eventos adversos. Em 2000, o relatório "To Err is Human: building a safer health system" desperta a atenção das instituições de saúde sobre eventos adversos que poderiam ser evitados nos Estados Unidos da América ${ }^{(1)}$. Diante dos resultados alarmantes, o tema ganhou relevância e em 2004 foi criada uma Aliança Mundial para a Segurança do Paciente que objetivou organizar os conceitos e definições sobre segurança do paciente e propor medidas para reduzir os riscos e eventos adversos ${ }^{(2)}$.

No Brasil, o Ministério da Saúde, por meio da Portaria № 529 de 1 으 de Abril de 2013, institui o Programa Nacional de Segurança do Paciente, com intuito de contribuir para a qualificação do cuidado em todos os estabelecimentos de saúde do território nacional e define segurança do paciente como redução, a um mínimo aceitável, do risco de dano desnecessário associado ao cuidado de saúde ${ }^{(3)}$. A partir de então foram traçadas ações para reduzir os riscos e difundiu-se o processo de acreditação em unidades de saúde, como por exemplo, em nível internacional a Joint Comission on Acreditation of Health care Organizations ${ }^{(4)}$, que se baseia nas seis Metas Internacionais de Segurança do Paciente.

Das seis metas elencadas, as instituições de saúde encontram dificuldades na comunicação segura como cultura a ser adotada para redução de eventos adversos relacionados à assistência. Comunicação efetiva pode melhorar o fluxo de informações e intervenções, aumentar a segurança, satisfação do paciente e diminuir o tempo de permanência hospitalar ${ }^{(4)}$.

A construção e aplicação de protocolos que visam a avaliação mais global das situações vivenciadas pelos profissionais, bem como programas de treinamento e educação adequados e eficazes, tornam a comunicação mais eficaz nas organizações de saúde, o que melhora a segurança e aumenta a satisfação do paciente. A crescente importância da satisfação do paciente e exigidas pelos credenciamentos estão causando uma mudança na forma como os hospitais avaliam e gerenciam suas organizações de saúde ${ }^{(5)}$.

Comunicaçãoe segurança do pacienteassumem particular importância em situações de transição do cuidado, como alta hospitalar. A alta hospitalar traz ao paciente, sentimentos ambíguos, tais como satisfação e medo. Satisfação pela recuperação e retorno para casa, e medo por sentir-se sem o suporte da equipe de saúde, ao considerar que a continuidade dos cuidados será realizada em domicílio(b). A adequada orientação ao paciente em alta hospitalar é necessária para prevenir, reduzir e detectar os riscos de eventos adversos ${ }^{(7)}$.

A relevância deste estudo está na originalidade da proposta de construção e validação de um instrumento que utiliza como indicador o processo de comunicação centrada na percepção do paciente. Ao considerar a necessidade de instrumentos para avaliação da compreensão do paciente sobre orientações de alta, o presente estudo teve como objetivo construir e investigar as propriedades psicométricas da escala "Avaliação da compreensão do paciente sobre orientações de alta".

\section{MÉTODO}

\section{Tipo de estudo}

Trata-se de um estudo metodológico quantitativo constituído por duas partes: construção de instrumento para a avaliação da compreensão do paciente sobre as orientações de alta e validação deste instrumento.

\section{Participantes da pesquisa}

O comitê de juízes foi composto por quatro enfermeiros, dois professores e dois leigos, totalizando oito participantes. O público alvo foi constituído por 20 pessoas: 10 pacientes e 10 enfermeiros.

O cálculo da amostra mínima para avaliação das propriedades psicométricas do instrumento foi obtido adotando o critério preconizado de cinco participantes por item da parte I escala elaborada ${ }^{(10)}$ e foi estabelecido mínimo de 70 pacientes.

Os critérios de inclusão foram pacientes internados nas unidades de internação eleitas para o estudo, adultos com idade superior a 18 anos de ambos os sexos e escolaridade mínima completa, 50 ano do ensino fundamental. A escolaridade mínima foi proposta pelas próprias características do instrumento, por tratar-se de uma avaliação da compreensão por meio de um instrumento escrito e pela escolha dos autores de mensurar o tempo de preenchimento da escala pelo paciente como um indicador de compreensão ou possibilidade de dúvida. Foram excluidos pacientes com diagnóstico de sindrome demencial ou transtornos psiquiátricos.

Participaram da etapa de avaliação psicométrica do instrumento também dois observadores. Um dos observadores foi o pesquisador, o outro foi uma enfermeira capacitada na decodificação dos sinais não verbais e que não atuava no hospital selecionado. Os dois observadores responderam simultaneamente a parte II da escala (compreensão percebida) e avaliaram a frequência de sinais não-verbais dos pacientes enquanto os mesmos respondiam a parte I (compreensão referida). 


\section{Local do estudo}

O estudo foi realizado em unidades de internação de um hospital filantrópico de São Paulo.

\section{Coleta de dados}

A coleta de dados ocorreu de fevereiro a dezembro de 2015. O processo de construção e validação do instrumento baseou-se no modelo proposto por Pasquali(8), composto por três etapas: procedimentos teóricos, procedimentos empíricos e procedimentos analíticos.

$\mathrm{Na}$ fase de procedimentos teóricos, são descritas a construção, validação aparentee de conteúdo do instrumento. A finalidade da escala é mensurar o processo de comunicação profissional-paciente, por meio da compreensão do paciente às orientações de alta hospitalar, e proporcionar maior segurança para o paciente na alta hospitalar. Entende-se que ao receber a alta hospitalar o paciente compreenda o seu tratamento e tenha elucidado as dúvidas referidas ou percebidas. Ao considerar que as orientações de alta hospitalar podem ser realizadas durante o tratamento, em um processo contínuo, a escala não se limita a avaliação de um profissional em um momento específico. Sendo assim, tem-se como finalidade a avaliação de um processo de comunicação e não exatamente de uma relação interpessoal.

A escala foi construida pelos autores, em 2013, devido a necessidade da avaliação tanto da comunicação verbal (referida) quanto não verbal (percebida) no processo de compreensão dos pacientes nas interações com a equipe de saúde durante situações de alta hospitalar, ao reconhecer que a comunicação efetiva é tão importante para adesão ao tratamento e prevenção de iatrogenias.

A revisão inicial da literatura sobre o tema foi realizada na plataforma Pubmed (2013), com a sintaxe ("Patient Discharge"[Mesh] AND "Validation Studies" [Publication Type] AND treatment information), na qual identificou-se 51 artigos. Esses artigos mostraram que há instrumentos disponiveis para mensurar satisfação dos pacientes com os serviços hospitalares, identificar eventos adversos, complicações pós-alta, fatores de risco, necessidade de aprendizagem de pacientes e cuidadores, risco de readmissão precoce, percepção do paciente sobre fatores cotidianos que afetam o cuidado pós alta, avaliação do conteúdo das anotações e planejamento do processo de alta. No entanto, não foram identificados instrumentos para a avaliação da compreensão do paciente sobre as orientações de alta, mostrando a lacuna de conhecimento neste tema.

Os referenciais teóricos utilizados na construção da escala "Avaliação da compreensão do paciente sobre orientações de alta" foram o livro "Comunicação tem remédio: a comunicação nas relações interpessoais em saúde"(7) e o Programa Nacional de Segurança do Paciente ${ }^{(3)}$. Os pressupostos teóricos eleitos para a construção da escala foram os sinais não-verbais que representam a compreensão ou a dúvida do paciente, e as informações necessárias e que devem ser compartilhadas na relação profissional-paciente no processo de alta hospitalar para assegurar a segurança do paciente neste processo.

A escala foi construida em duas partes: a parte I e II. A parte I refere-se à compreensão referida pelo paciente sobre as orientações de alta, foi respondida pelo paciente e possui 14 itens. Foi utilizada escala tipo Likert e os graus variados de concordância, neste estudo, foram os seguintes: 1- não recebi orientação, 2- discordo, 3- discordo parcialmente, 4concordo parcialmente e 5- concordo.

A parte II refere-se à compreensão percebida em que os indicadores são os sinais não verbais do paciente. Foi respondida por um observador enfermeiro e continha oito itens. Foi utilizada também uma escala tipo Likert e os graus variados de frequência, neste estudo, foram: 1- nunca, 2quase nunca, 3- às vezes, 4- quase sempre e 5- sempre.

Na parte II, o observador avalia a frequência de sinais não verbais do paciente durante o preenchimento da primeira parte do instrumento, bem como, controla o tempo de preenchimento da parte I. A parte II contém pictogramas, simbolos que representam um objeto ou um conceito por meio de desenhos figurativos, para facilitar a identificação dos sinais não verbais. Outro indicador utilizado foi a identificação do item em que o paciente teve maior hesitação em responder. Após a observação de hesitação, o profissional teve a oportunidade de identificar as possiveis dúvidas e registrar o que deve ser orientado novamente.

A escala tem quatro itens negativos (016, O17, O19 e Q21) que requerem codificação reversa antes do cálculo do escore. Após a recodificação destes itens, o cálculo do escore de cada fator e de cada parte do instrumento é obtido por uma somatória simples. Por fim, o escore final de cada fator (são 4 fatores) e de cada parte do instrumento (parte I e II) é obtido pelo re-escalonamento de 0 a 100 para torná-los comparáveis. Sendo assim, quanto maior o escore, melhor é a compreensão do paciente em relação ao seu tratamento e alta.

$\mathrm{Na}$ fase dos procedimentos teóricos, os juízes foram convidados por e-mail, receberam pessoalmente o questionário após agendamento e foram orientados a verificar a equivalência semântica, conceitual e objetividade dos itens.

O questionário aplicado aos juízes possibilita concordar ou discordar de cada uma das partes que compõe a escala (título, objetivos, instruções gerais, instruções para o preenchimento da parte I, itens da parte I, respostas psicométricas tipo Likert da parte I, instruções para o preenchimento da parte II, itens da parte II, respostas psicométricas tipo Likert da parte 
II, pictogramas ilustrativos). Foram questionados também em relação a alguma dificuldade de compreensão em algum item da escala, se tiveram que ler mais de uma vez algum item e se recomendariam a exclusão de algum item. Em caso de resposta afirmativa, eles precisam descrever a sugestão. $O$ nível de concordância considerado como aceitável para os itens analisados foi de $80 \%$ e baseou-se no Índice de Validade de Conteúdo(8-9)

Ainda na fase dos procedimentos teóricos, o instrumento foi aplicado no público alvo por conveniência. A escala foi aplicada de forma individual para que cada participante relatasse suas dificuldades e facilidades no seu preenchimento, e sugerisse modificações na redação dos itens, caso necessário. Por fim, foi realizada uma revisão do processo de validação de conteúdo e os pesquisadores procederam adequações necessárias elaborando a versão para fase de procedimentos empíricos.

$\mathrm{Na}$ fase dos procedimentos empíricos, os pacientes foram abordados no quarto/enfermaria onde se estavam internados enquanto o acompanhante foi entregar a documentação referente à alta no setor de internação para registro da saída do hospital. Isso levava em torno de 10 minutos e permitiu a realização da pesquisa sem transtornos para o paciente. Após o convite para participar do estudo, para aqueles que concordaram, foi solicitado sentar-se na poltrona situada dentro do quarto e oferecida uma prancheta com a escala para responder os itens sobre a caracterização da amostra e a parte I da escala.

Os pacientes receberam instruções de marcar a resposta que melhor reflete a sua compreensão das orientações de alta durante toda sua internação e assinalasse somente uma alternativa em cada item. Também foi solicitado não deixar nenhum item em branco e não conversar com os observadores durante o preenchimento. As dúvidas deveriam ser esclarecidas após o preenchimento, para assegurar a acurácia das informações. O paciente foi instruido que "não ter medicação", "não ter restrição física ou alimentar" ou "não ter cuidados especiais e/ou procedimentos" são consideradas orientações. Além disso, os pacientes tinham a oportunidade de redigir por extenso outras dúvidas em relação às orientações de alta.

Os observadores leram atentamente os itens antes de preencher a parte II da escala e mantiveram uma distância mínima de aproximadamente 2 passos largos do paciente, em uma posição que possibilitasse a visualização da face do paciente e foram orientados a não fixar o olhar na face do paciente por um tempo prolongado. Essa recomendação teve como finalidade diminuir a probabilidade do paciente sentir-se invadido no espaço pessoal, além de permitir a identificação por parte do observador a compreensão percebida do item em que o paciente teve maior hesitação em responder.

Também receberam outras orientações, tais como, o profissional que realizou a última orientação não deveria ser o mesmo que fosse realizar a observação para evitar o viés de constrangimento do paciente em assumir que não compreendeu as orientações imediatas. Ao identificar incompreensão referida ou percebida, o profissional deveria, por uma questão ética, esclarecer as dúvidas do paciente. O observador avaliou a frequência de sinais não verbais do paciente durante o preenchimento da parte II do instrumento, bem como, controlou o tempo de preenchimento da parte I.

\section{Procedimentos de análise dos dados}

$\mathrm{Na}$ fase dos procedimentos analíticos, os dados foram analisados por meio da análise descritiva e inferencial com o uso do software SPSS (Statistical Package for the Social Sciences) versão 20.0. Para as variáveis categóricas, foram apresentadas as frequências absolutas e relativas, e para as variáveis numéricas, medidas-resumo (média, quartis, mínimo, máximo e desvio padrão). As associações lineares entre duas variáveis numéricas foram avaliadas por Correlação de Pearson

Para fins estatísticos, as respostas ao item "Não recebeu orientação" foram desconsideradas apenas da análise do processo de validação, pois a finalidade do instrumento é avaliar a compreensão sobre as orientações de alta; entretanto essa resposta não deve ser excluída na aplicação do instrumento, pois representa um item sem orientação, ou seja, um dado de pesquisa.

Procedeu-se à avaliação da dimensionalidade utilizandose a análise fatorial pelo método por componentes principais e rotação ortogonal VARIMAX. A concordância interobservadores do escore total foi avaliada via correlação intraclasse. Para todos os testes estatísticos foram adotados um nivel de significância de 5\%. Em seguida, foram calculados os coeficientes Alpha de Cronbach para se avaliar a consistência interna dos itens que compunham cada fator. De acordo com George e Mallery ${ }^{(11)}$, Alpha de Cronbach acima de 0,9 é considerado excelente; de 0,8 a 0,9 bom; de 0,7 a 0,8 aceitável de 0,5 a 0,7 pobre; e abaixo de 0,5 inaceitável.

\section{Procedimentos éticos}

A realização desta pesquisa atendeu às normas nacionais e internacionais de ética em pesquisa envolvendo seres humanos: Declaração de Helsinki(12) e Resolução Nacional 466/2012(13) , foi aprovada pelo Comitê de Ética e Pesquisa da instituição co-participante (número de parecer 1.001.679) e todos os participantes assinaram o Termo de Consentimento Livre e Esclarecido. 


\section{RESULTADOS}

Em relação à avaliação dos juizes, os resultados mostraram que houve avaliação positiva da escala, bem como a relevância da construção deste instrumento de medida. Foi necessária apenas uma rodada de validação para atingir o indice de concordância. Dentre sugestões propostas, apenas uma não foi acatada pelos autores e foi incluído um item na parte I da escala, denominado "Compreendi cuidados especiais e/ou procedimentos que devo realizar após minha alta (ex.: curativo, sonda nasoenteral, sonda vesical de demora, oxigenioterapia.... No item "Compreendi os horários diários (ex.: 6h, 14h, 22h) e os intervalos (ex.: 4/4h, 8/8h, 12h/12h) em que devo tomar cada uma das minhas medicações", foram suprimidas as informações entre parênteses e uma palavra foi substituída nas instruções da parte I da escala, "não interaja" por "não converse".

Um dos juízes sugeriu a troca da escala tipo Likert de concordância para uma tipo Likert de frequência, que contemplassem as respostas: não recebi orientação, recebi pouca orientação, recebi as orientações necessárias, estou plenamente orientado e satisfeito para a alta. Essa sugestão não foi atendida, pois o objetivo da escala é avaliar a compreensão e não a frequência de orientação. Os resultados do pré-teste com o público alvo mostraram que os itens da escala estavam adequados e não houve sugestões para alterações.

Para obtenção do perfil psicométrico, a escala "Avaliação da compreensão do paciente sobre orientações de alta" foi aplicada em uma amostra de 72 pacientes e dois observadores. A média de idade dos pacientes foi de 43,1 anos $( \pm 12,6)$, tempo médio de internação médio 10,7 dias $( \pm 5,7)$, a maior parte dos diagnósticos médicos referia-se ao sistema cardiovascular $(n=15 ; 20,8 \%)$, seguido do sistema digestório ( $n=14 ; 19,4 \%)$ e a maioria dos pacientes recebeu a última orientação por enfermeiros ( $n=52 ; 72,2 \%)$.
Os resultados mostraram que houve participação similar de homens e mulheres ( $p=0,480$ - Teste de Qui-Quadrado), sendo $54,2 \%$ do sexo masculino e $45,8 \%$ do feminino. A maioria é natural do estado de São Paulo ( $n=48 ; 66,7 \%)$, metade dos pacientes eram católicos ( $n=36 ; 50 \%)$ e a maior frequência foi de pacientes casados ( $n=34 ; 47,2 \%)$ e que possuíam até o ensino fundamental 2 completo ( $n=31 ; 43,1 \%)$.

A análise fatorial da parte I resultou em quatro fatores que explicam $77,9 \%$ da variância total dos dados. A escolha deste número de fatores deu-se a partir do número de autovalores da matriz de correlação maiores do que um. Um autovalor pequeno sugere uma pequena contribuição do fator na explicação na variação das variáveis originais. Os resultados da análise fatorial foram interpretados por meio das "cargas fatoriais" em que cada uma representa a medida de correlação entre o fator derivado da análise e os itens originais (Tabela 1).

A análise fatorial permite a decomposição da variância de cada item em duas partes: parte comum e parte específica. A parcela da variância comum ocorre devido a fatores comuns e é denominada de comunalidade. Dessa forma, itens cuja variância devido a fatores comuns for inferior a 50\% devem ser excluídos por estarem pouco representados na análise fatorial, o que não aconteceu com nenhum item da escala(10) (Tabela 1).

O fator 1 foi nomeado "compreensão sobre medicamentos" e é formado por quatro itens ( $0.3,0.4,0.5$ e Q.6). O fator 2, nomeado "compreensão sobre tratamento e diagnóstico", é composto por cinco itens (Q.1, Q.2, Q.12, Q.13 e Q.14). O Fator 3 foi nomeado "compreensão sobre restrição" e é formado por três itens (0.9, Q.10 e Q.11). O último fator, o fator 4, foi nomeado "compreensão sobre retorno" e é formado apenas por dois itens (0.7 e 0.8). Valores altos obtidos nestes fatores, apontam concordância em relação aos itens (Tabela 1).

Tabela 1 - Cargas fatoriais rotacionadas pela transformação VARIMAX e comunalidades dos itens da parte I da escala "Avaliação da compreensão do paciente sobre orientações de alta". São Paulo, SP, 2015.

\begin{tabular}{|c|l|l|l|c|}
\hline \multicolumn{4}{|c|}{ Fatores } & \multirow{2}{*}{$\begin{array}{c}\text { Comunali- } \\
\text { dades }\end{array}$} \\
\cline { 1 - 2 } 1 & 2 & 3 & 4 & \\
\hline
\end{tabular}

Q.5 Compreendi até quando devo tomar ou aplicar cada uma das minhas medicações.

Q.4 Compreendi os horários diários e os intervalos em que devo tomar cada uma das minhas medicações.

O.3 Compreendi quantas medicações precisarei tomar ou aplicar.

Q.6 Compreendi para que serve cada uma das minhas medicações, ou seja, o benefício de tomar cada uma delas.

\begin{tabular}{c|ccccc}
0,885 & 0,206 & 0,158 & 0,095 & 0,860 \\
\hline 0,857 & 0,332 & 0,199 & 0,000 & 0,884 \\
\hline 0,839 & 0,377 & 0,142 & 0,111 & 0,879 \\
\hline 0,776 & 0,209 & 0,191 & 0,184 & 0,716
\end{tabular}


Q.13 Compreendi as orientações sobre o meu tratamento.

\begin{tabular}{|c|c|c|c|c|}
\hline 0,177 & 0,758 & 0,247 & 0,106 & 0,678 \\
\hline 0,376 & 0,754 & 0,165 & 0,092 & 0,745 \\
\hline 0,235 & 0,741 & 0,261 & 0,128 & 0,688 \\
\hline 0,394 & 0,728 & 0,136 & 0,149 & 0,726 \\
\hline 0,197 & 0,719 & 0,387 & 0,101 & 0,717 \\
\hline 0,116 & 0,231 & 0,854 & 0,176 & 0,827 \\
\hline 0,238 & 0,242 & 0,828 & 0,015 & 0,802 \\
\hline 0,213 & 0,338 & 0,803 & 0,036 & 0,806 \\
\hline 0,097 & 0,140 & $-0,078$ & 0,887 & 0,823 \\
\hline 0,143 & 0,146 & 0,320 & 0,783 & 0,757 \\
\hline 3,39 & 3,34 & 2,61 & 1,56 & \\
\hline 0,919 & 0,868 & 0,884 & 0,650 & \\
\hline
\end{tabular}

O.2 Saberei explicar para os outros o meu problema de saúde.

\section{ARTICO 20}

O.14 Fui esclarecido em minhas dúvidas

O.1 Compreendi o meu diagnóstico médico.

O.12 Compreendi o período total do meu tratamento, ou seja, até quando devo seguir as orientações dadas.

Q.10 Compreendi a necessidade de restrição ou estímulo à atividade física no meu tratamento.

O.ll Compreendi cuidados especiais e/ou procedimentos que devo realizar após minha alta

Q.9 Compreendi cuidados que devo ter com a minha alimentação diária.

Q.7 Compreendi se é preciso e quando deve ser o meu retorno para acompanhamento.

O.8 Compreendi quais os sinais de piora da minha doença, ou seja, quando devo procurar um serviço de emergência.

Autovalores

Alpha de Cronbach

$\mathrm{N}=70$. A medida de Kaiser-Meyer-Olkin (KMO) verificou a adequação amostral para a análise (KMO=0,779). O teste de esfericidade de Bartlett [Qui-quadrado $(91)=762,66$, $(p<0,001)]$, indicou que as correlações entre os itens são suficientes para a realização da análise. Nenhum autovalor foi menor que 1 , portanto nenhum fator deve ser excluido. Fonte: elaboração própria com base nos dados obtidos no estudo.

Verificou-se consistência interna excelente quanto ao fator "compreensão sobre medicamentos" $(\alpha=0,919)$ e boa relacionada aos fatores "compreensão sobre tratamento e diagnóstico" ( $\alpha=0,868)$, e "compreensão sobre restrição" $(\alpha=0,884)$. Exceto o fator referente à "compreensão sobre retorno" ( $\alpha=0,650)$ apresentou moderada consistência interna. O valor de Alpha de Cronbach global para a parte I da escala foi considerada excelente $(\alpha=0,918)$.

Para a parte II da escala, procedeu-se a análise fatorial pelo método por componentes principais e rotação ortogonal VARIMAX, entretanto estabeleceu-se uma fraca interdependência entre as variáveis, não sendo possível identificar fatores com um grau razoável de interpretação. Portanto, optou-se pelo uso da parte II como um fator único.

Na parte II, verificou-se consistências internas aceitáveis, tanto para observador $1(\alpha=0,724)$ quanto para o observador $2(\alpha=0,749)$. Nota-se ainda que, apesar dos itens 0.17 e 0.22 apresentarem correlações item-total valores inferiores a 0,30 (correlação fraca), a exclusão desses itens acarreta uma pequena elevação na consistência interna. Por isso, nenhum item é sugestivo de eliminação (Tabela 2).
Tabela 2 - Correlação de cada item com o escore total do instrumento, Alpha de Cronbach global se o item for excluido na parte II da escala "Avaliação da compreensão do paciente sobre orientações de alta”. São Paulo, SP, 2015.

\begin{tabular}{|l|c|c|}
\hline Itens & $\begin{array}{c}\text { Correlação } \\
\text { Item - } \\
\text { total }\end{array}$ & $\begin{array}{c}\text { Alpha de } \\
\text { Cronbach } \\
\text { se o item } \\
\text { for excluido }\end{array}$ \\
\hline $\begin{array}{l}\text { Observador } 1 \text { (Alpha de Cronbach } \\
\text { global }=0.724)\end{array}$
\end{tabular}

O.15 Expressão facial sorridente, $\begin{array}{lll}\text { mostrando satisfação ou } & 0,356 & 0,707\end{array}$ compreensão.

Q.16 Expressão facial de dúvida, sobrancelhas franzidas ou boca cerrada.

$0,499 \quad 0,681$

O.17 Pára para pensar na resposta, olhando para cima ou direcionando o $\quad 0,183 \quad 0,745$ olhar para alguma direção.

O.18 Meneio positivo com a cabeça, ou $\quad 0,433 \quad 0,692$ seja, dar sim com a cabeça.

O.19 Meneio negativo com a cabeça, ou seja, dar não com a cabeça.

0,406

0,698

0.20 Postura relaxada, mas atenta.

0,623

0,649 
Q.21 Postura rígida, tensa ou mostrando preocupação.

$0,537 \quad 0,668$

O.22 Responde prontamente os itens.

0,315

0,715

Observador 2 (Alpha de Cronbach global

$=0.749$ )

Q.15 Expressão facial sorridente, mostrando satisfação ou compreensão.

O.16 Expressão facial de dúvida, sobrancelhas franzidas ou boca cerrada.

O.17 Pára para pensar na resposta,

olhando para cima ou direcionando o

olhar para alguma direção.

O.18 Meneio positivo com a cabeça, ou seja, dar sim com a cabeça.

O.19 Meneio negativo com a cabeça, ou seja, dar não com a cabeça.

0.20 Postura relaxada, mas atenta.

0.21 Postura rígida, tensa ou mostrando preocupação.

O.22 Responde prontamente os itens.

$\mathrm{N}=72$. Os itens $0.16,0.17,0.19$ e 0.21 tem codificação reversa.

A concordância entre os observadores na parte II do instrumento obteve um coeficiente de correlação Intraclasse $=0.824$ (IC95\%: 0,734-0,886) e apontou uma boa reprodutibilidade inter-observador. Corroborando com esse resultado, verificou-se uma forte correlação ( $r=0,826$, $p<0,001-$ Correlação de Pearson) entre os escores da parte II pontuados pelos observadores 1 e 2 .

Tanto para o escore do paciente como do observador, o escore bruto foi obtido via soma das pontuações atribuídas aos itens. Em seguida, para tornar todos os escores comparáveis, os escores brutos foram reescalonados de tal forma que variam de 0 a 100 pontos (Tabela 3).

Tabela 3 - Medidas-resumo dos escores da escala "Avaliação da compreensão do paciente sobre orientações de alta". São Paulo, SP, 2015.

\begin{tabular}{|c|c|c|c|c|c|c|}
\hline Escore & Média & $\begin{array}{l}\text { Desvio } \\
\text { Padrão }\end{array}$ & $\begin{array}{l}\text { Mini- } \\
\text { mo }\end{array}$ & Máximo & $\begin{array}{l}\text { Media- } \\
\text { na }\end{array}$ & $N$ \\
\hline \multicolumn{7}{|l|}{ Parte I - paciente } \\
\hline Total & 77,4 & 19,2 & 23,8 & 100,0 & 79,8 & 70 \\
\hline $\begin{array}{l}\text { Compreensão } \\
\text { sobre } \\
\text { medicamentos }\end{array}$ & 71,4 & 26,9 & 0,0 & 100,0 & 75,0 & 72 \\
\hline $\begin{array}{l}\text { Compreensão } \\
\text { sobre tratamento } \\
\text { e diagnóstico }\end{array}$ & 83,4 & 20,2 & 20,0 & 100,0 & 93,3 & 71 \\
\hline $\begin{array}{l}\text { Compreensão } \\
\text { sobre restrição }\end{array}$ & 69,0 & 29,9 & 0,0 & 100,0 & 77,8 & 71 \\
\hline $\begin{array}{l}\text { Compreensão } \\
\text { sobre retorno }\end{array}$ & 87,6 & 17,8 & 33,3 & 100,0 & 100,0 & 71 \\
\hline \multicolumn{7}{|l|}{$\begin{array}{l}\text { Parte II - } \\
\text { observador }\end{array}$} \\
\hline Total & 61,3 & 18,2 & 28,1 & 90,6 & 64,1 & 72 \\
\hline
\end{tabular}

Foram comparadas as percepções sobre compreensão da orientação de alta dos pacientes (total parte I) e dos observadores (total parte II), e observou-se uma correlação positiva entre os dois escores de compreensão $(r=0,439, p<0,001)$, entretanto fraca. Sendo assim, quanto maior a percepção de compreensão sobre orientação de alta referida pelos pacientes, mais os observadores, perceberam essa compreensão pela decodificação dos sinais não verbais.

Também, verificaram-se correlações fracas positivas entre os escores totais dos observadores (parte II) e os fatores "compreensão sobre medicação" ( $r=0,428, p<0,001)$, "compreensão sobre tratamento e diagnóstico" ( $r=0,421$, $p<0,001)$ e "compreensão sobre retorno" ( $r=0,254, p=0,032$ ). Não se verificou correlação significativa entre o escore total do observador (parte II) e o fator "compreensão sobre restrição" $(r=0,226, p=0,058)$.

Essas correlações estatisticamente significativas entre os sinais não verbais percebidos nos pacientes pelos observadores e a compreensão referida em relação a medicação, tratamento/ diagnóstico e as condições de retorno, reforçam a necessidade de os profissionais ficarem atentos para além da comunicação verbal dos pacientes, ou seja, os sinais não verbais são indicadores também seguros nas relações interpessoais.

O tempo médio de preenchimento da parte I da escala pelos pacientes foi de $5,9( \pm 1,9)$ minutos e o item percebido pelo observador com maior hesitação de resposta foi o 0.6 "Compreendi para que serve cada uma das minhas medicações, ou seja, o benefício de tomar cada uma delas", o que reforça falta de informação sobre os medicamentos.

\section{DISCUSSÃO}

Considerar o processo de comunicação na sua totalidade aumenta as chances do profissional ser mais assertivo. Do processo de interação interpessoal, apenas 7\% dos pensamentos são transmitidos por palavras ${ }^{(7)}$, ou seja, a valorização das palavras, por si só, é insuficiente para interpretar a compreensão do paciente. Entretanto, a correta decodificação e interpretação da comunicação não verbal requerem capacitação, adaptação e atenção constante, pois sinais não verbais são emitidos ao mesmo tempo da fala(14).

Confirmar o entendimento dos pacientes sobre o tratamento e diagnóstico é essencial para segurança. Validar a compreensão das orientações dadas é uma forma de identificar a necessidade de reorientação das ações educativas e planejamento dos cuidados $^{(15)}$

A falta de compreensão sobre para que servem as medicações e o benefício de tomá-las é séria e pode interferir na adesão do paciente ao tratamento e na sua segurança. Estudos mostram que pacientes, em geral, apresentam baixos níveis de conhecimento sobre seu tratamento, especialmente sobre medicamentos e, consequentemente baixa adesão ao tratamento(16-20). Outro estudo apontou que dois terços das informações prestadas pelos profissionais de saúde são esquecidas imediatamente e $50 \%$ das informação que parece ter sido memorizada é recordada de forma errada pelos pacientes ${ }^{(21)}$. 
Promover a compreensão do paciente sobre sua condição de saúde-doença e/ou tratamento exige que o profissional de saúde ressignifique o cuidar nas diferentes propositivas técnicas e políticas para a ampliação das práticas seguras. Além disso, é importante o profissional se capacitar cientificamente e comprometer-se eticamente com ações sistêmicas de avaliação e prevenção, para com isso viabilizar a redução de desfechos indesejados e analisar, criteriosamente, o impacto das práticas seguras sobre a qualidade do cuidado(22).

\section{Limitações do estudo}

Uma limitação encontrada no estudo, refere-se à exclusão dos individuos analfabetos ou com baixa escolaridade. Por outro lado, a inclusão desses indivíduos no presente estudo poderia trazer o risco em relação a respostas imprecisas e pouco confiáveis, devido às características do instrumento que exigem leitura e compreensão. Portanto, estudos futuros são necessários para avaliação da validade deste instrumento em pacientes analfabetos ou com baixa escolaridade.

\section{Contribuição do estudo para a prática}

Este estudo contribui para a prática de pesquisadores e profissionais da área de saúde na melhoria da comunicação e segurança do paciente no processo de alta hospitalar. Acreditase que a construção e a validação de um instrumento baseado na avaliação das compreensões referidas e percebidas sobre as orientações de alta poderá aprimorar as estratégias e intervenções durante esse processo.

\section{CONCLUSÃO}

A escala "Avaliação da compreensão do paciente sobre orientações de alta" é um instrumento confiável e válido para avaliar as orientações dadas por profissionais de saúde aos pacientes sobre o seu tratamento pós-alta. Correlações existentes entre os sinais não verbais e a compreensão referida reforçam a importância e necessidade do uso da parte II da escala. A parte I (compreensão referida) e a parte II (compreensão percebida) são complementares e devem ser utilizadas como um instrumento único.

Contribuição dos autores: Rednaj Jaure Bacury Souza Mota: coleta de dado, análise e interpretação dos dados, redação do artigo e revisão final. Ana Claudia Puggina: concepção e desenho, análise e interpretação dos dados, redação do artigo, revisão crítica e revisão final.

\section{REFERÊNCIAS}

1. Kohn L, Corrigan J, Donaldson M. To err is human: building a safer heath system. Washington, DC: Committee on Quality of Health Care in America. Institute of Medicine: National Academy Press: 2000.

2. World Health Organization (WHO). World Alliance for Patient Safety. Forward Programme 2008-2009. Geneva (Swi): World Health Organization; 2010.

3. Ministério da Saúde (BR). Portaria no. 529, de 1 de abril de 2013. Institui o Programa Nacional de Segurança do Paciente (PNSP). Diário Oficial da União 01 abr 2013:Seção 1

4 . Joint Commission International. Joint commission international accreditation standards for hospitals. 6th ed. Estados Unidos: Joint Commission Resources; 2017.

5. Burgener AM. Enhancing communication to improve patient safety and to increase patient satisfaction. Health Care Manag (Frederick) [Internet]. 2017 [cited 2019 Nov 23];36(3):238-43. Available from: http://dx.doi.org/10.1097/ HCM.0000000000000165

6. Pompeo DA, Pinto MH, Cesarino CB, Araújo RRDF, Poletti NAA. Atuação do enfermeiro na alta hospitalar: reflexões a partir dos relatos de pacientes. Acta Pau Enferm [Internet]. 2007 [cited 2016 Mai 16]:20(3):345-50. Available from: http://dx. doi.org/10.1590/S0103-21002007000300017

7. Silva MJP. Comunicação tem remédio: a comunicação nas relações interpessoais em saúde. 9ạ ed. São Paulo: Loyola, 2013.

8 .Pasquali L. Psicometria - Teoria dos testes na psicologia e educação. 5a ed. Petrópolis (RJ): Vozes, 2013.

9 .Hyrkäs K, Appelqvist-Schmidlechner K, Oksa L. Validating an instrument for clinical supervision using an expert panel. Int J Nurs Stud [Internet]. 2003 [cited 2019 Nov 23]; 40(6):619-25. Available from: https://doi.org/10.1016/S00207489(03)00036-1

10. Hair JF Jr, Black WC, Babin BJ, Anderson RE, Tatham RL. Análise multivariada de dados. 6 a ed. Porto Alegre: Bookman, 2009

11. George D, Mallery P. IBM SPSS Statistics 23 step by step: A simple guide and reference. 14th ed. New York: Routledge; 2016.

12. Asociación Médica Mundial. Declaración de Helsinki de la Asociación Médica Mundial. Principios éticos para las investigaciones médicas en seres humanos. Fortaleza: 64.a Asamblea General de la AMM; 2013 [cited 2019 Mar 14]. Available from: https://goo.gl/hvf7l

13. Ministério da Saúde (BR). Conselho Nacional de Saúde. Resolução n. 466, de
12 de dezembro de 2012. Aprova diretrizes e normas regulamentadoras de pesquisas envolvendo seres humanos. Brasilia, Diário Oficial da União, 12 dez. 2012. [cited 2019 Mar 14]. Available from: http://bvsms.saude.gov.br/bvs/saudelegis/ cns/2013/res0466_12_12_2012.htm.

14. Knapp ML, Hall JA, Horgan, TG. Nonverbal communication in human interaction. 8th ed. Boston: Cengage Learning, 2013.

15. Haas L, Maryniuk M, Beck J, Cox CE, Duker P. Edwards L, et al. National Standards for Diabetes Self-Management Education and Support. Diabetes Care [Internet]. 2014 [cited 2016 Feb 16];37(Suppl 1):S144-S153. Available from: https://doi. org/10.2337/dc12-1707

16. Sgnaolin V. Figueiredo AEPL. Adesão ao tratamento farmacológico de pacientes em hemodiálise. J Bras Nefrol [Internet]. 2012 [cited 2016 Feb 20]:34(2):109-16. Available from: http://dx.doi.org/10.1590/s0101-28002012000200002

18. Chan FW, Wong FY, So WY, Kung K, Wong CK. How much do elders with chronic conditions know about their medications? BMC Geriatrics [Internet]. 2013 [cited 2016 Feb 22]:13:59. Available from: https://doi.org/10.1186/1471-2318-13-59

19. Martins K, Macedo-Costa KN, Oliveira DS, Valdevino S, Rezende LC, Costa T. Nurse's role on preparing for discharge of surgical patients. Revista de Pesquisa: Cuidado é Fundamental Online [Internet]. 2015 [cited 2016 Mar 01]]7(1), 1756-1764. Available from: http://dx.doi.org/10.9789/2175-5361.2015.v7i1.1756-1764

20. Remondi FA, Cabrera MAS, Souza RKT. Não adesão ao tratamento medicamentoso continuo: prevalência e determinantes em adultos de 40 anos e mais. Cad Saúde Pública [Internet]. 2014 [cited 2016 Mar 03];30(1):126-136. Available from: http://dx.doi.org/10.1590/0102-311X00092613

21. Tavares NUL, Bertoldi AD, Mengue SS, Arrais PSD, Luiza VL, Oliveira MA, et al Factors associated with low adherence to medicine treatment for chronic diseases in Brazil. Rev Saúde Públ [Internet]. 2016 [cited 2016 Abr 16];50(Suppl 2):10s. Available from: $h$ ttp://dx.doi.org/10.1590/s1518-8787.2016050006150

22. Sathvik BS, Mangasuli S, Narahari MG, Gurudev KC, Parthasarathi G. Medication knowledge of hemodialysis patients and influence of clinical pharmacist provided education on their education. Indian J Pharm Sci [Internet]. 2007 [cited 2016 May 16]:62(2):232-9. Available from: http://dx.doi.org/10.4103/0250-474X.33149

23. Vargas MA, Luz AMH. Práticas seguras do/no cuidado de enfermagem no contexto hospitalar: é preciso pensar sobre isso e aquilo. Enferm foco [Internet]. 2010 [cited 2019 Nov 23]:1(1):23-7. Available from: http://revista.cofen.gov.br/index.php/ enfermagem/article/view/5 